\title{
LA AUDIENCIA COMO AUTOR: NARRATIVAS TRANSMEDIA Y PROPIEDAD INTELECTUAL DEL PÚBLICO. ALGUNAS REFLEXIONES JURÍDICAS
}

\author{
Javier Díaz-Noci y Anna Tous-Rovirosa
}

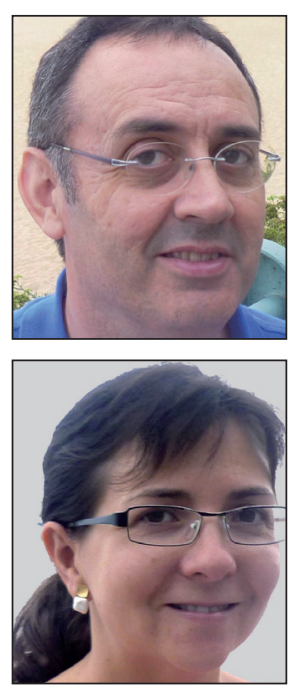

Javier Díaz-Noci es catedrático de la Universitat Pompeu Fabra de Barcelona, en la cual dirige el Máster de Estudios Avanzados en Comunicación Social. Profesor visitante en la University of Oxford (1998-1999). Diploma de estudios avanzados en derecho por la Universidad del País Vasco, autor de artículos y libros sobre derecho de autor, entre los que se incluyen Los resúmenes de prensa en los gabinetes de comunicación: Una aproximación jurídica (2004). Ha coordinado grupos de investigación competitivos y una red de investigadores España-Brasil de análisis comparado de cibermedios.
Universidad Pompeu Fabra, Facultad de Comunicación Roc Boronat, 138. 08018 Barcelona javier.diaz@upf.edu

Anna Tous-Rovirosa es profesora lectora del Depto. de Periodismo y Ciencias de la Comunicación de la Universidad Autónoma de Barcelona. Su tesis doctoral (UAB, 2008)-que mereció el II Premio CAC a la Investigación en Comunicación-, su libro La era del drama (UOC Press, 2010) y varios de sus artículos en publicaciones científicas versan sobre las series estadounidenses y su auge, así como las estrategias de interactividad y las nuevas relaciones que se establecen entre productores y consumidores. $\mathrm{Ha}$ sido profesora de la Univ. Federal de Bahía (Brasil) y de la Univ. del Rühr-Bochum (Alemania).

Universidad Autónoma de Barcelona (UAB), Facultat de Ciències de la Comunicació Bellaterra, Cerdanyola del Vallès, 08193 Barcelona anna.tous@uab.cat

\section{Resumen}

Desde que se configuró en el ordenamiento jurídico a principios del siglo XVIII en Inglaterra, el derecho de autor se enfrenta con cada nueva tecnología a una necesaria adaptación de sus mecanismos de protección a los autores. Las herramientas de edición y difusión de internet han potenciado fenómenos que ya existían, como la participación del público o la extensión narrativa a través de la transformación de obras ya existentes, y han hecho que los usuarios se conviertan, de una u otra manera, en autores. Fenómenos como el periodismo participativo o ciudadano o las fanfictions, que además se manifiestan en diversos medios, lo que se ha dado en llamar narrativas transmediáticas, han provocado que el derecho se ocupe de ellas. Este texto analiza los nuevos retos que dicha narrativa ha planteado al derecho de autor.

\section{Palabras clave}

Propiedad intelectual, Derecho de autor, Narrativa transmediática, Fanfiction, Periodismo participativo, Cibermedios.

Title: The audience as author: transmedia narrative and intellectual property. Some juridical reflexions

\begin{abstract}
Since copyright first appeared in the legal system in the 17th century, with every new technology the enforcement of authors' rights has faced a necessary adaptation of the mechanisms to protect authors. The popularization of the Internet's publishing and dissemination tools has multiplied phenomena which previously existed, such as reader participation or narrative extension through transformation of preexisting works. In one way or another, this promotes the audience to the category of (co)authors. Participative or citizen journalism and fanfictions, which have appeared in several media, have produced the so-called transmedia narratives, which present certain legal considerations. This text analyzes the challenges that transmedia narrative has posed to the intellectual property system.
\end{abstract}

\section{Keywords}

Intellectual property, Authors' rights, Transmedia narrative, Fanfiction, Participative journalism, Online media.

Díaz-Noci, Javier; Tous-Rovirosa, Anna. "La audiencia como autor: narrativas transmedia y propiedad intelectual del público. Algunas reflexiones jurídicas". El profesional de la información, 2012, septiembre-octubre, v. 21, n. 5, pp. $458-467$.

http://dx.doi.org/10.3145/epi.2012.sep.03

Artículo recibido el 29-06-12

Aceptación definitiva: 06-09-12 


\section{Narrativas participativas}

La narrativa es el género más presente en nuestra vida cotidiana. En cualquiera de sus variedades (prensa, radio, televisión, cine, literatura) los medios nos ofrecen múltiples productos narrativos, de ficción y de no ficción. Desde al menos la invención de la imprenta, tales productos siempre tenían unos autores y se dirigían a un público determinado. Sin embargo en la actualidad se producen trabajos con múltiples autores, o derivados de otros anteriores, que se difunden por distintos medios. A nivel de investigación, obras de autores como Mieke Bal o el Living handbook of narratology, del Interdisciplinary Center for Narratology de la Universidad de Hamburgo, se han combinado con las aportaciones de teóricos del hipertexto como Espen Aarseth, para dar como resultado trabajos como los de Liv Hausken (2004, pp. 395-396) o Marie-Laure Ryan y sus seguidores (Passalacqua; Pianzola, 2011), que investigan las propiedades de la narratología en los textos interactivos. El carácter multimedia y multimodal de muchos de esos relatos ha llevado a hablar de narrativa transmediática (Ryan, 2004). "El carácter polifacético de la narración no es nada nuevo", explica Ruth Page, pero analizarla en toda su complejidad "significa que las historias de las que nos ocupamos son de muchos más tipos que los textos literarios típicos de la narratología clásica" (Page, 2010, p. 11). Los contenidos producidos por los usuarios contribuyen a este carácter narrativamente innovador de los nuevos medios.

Si bien no se trata de fenómenos estrictamente nuevos [cartas al director en la prensa, llamadas del público en programas de televisión o radio, adaptación de novelas al cine, conversión de películas en series de televisión (Evans, 2011), o en comics, son prácticas habituales], la popularización de la Web en la última década del siglo XX y lo que llevamos del XXI y la facilidad para editar y publicar mediante herramientas digitales en una red de hipertexto global han incrementado la participación activa del público y la reivindicación por parte de los usuarios de su condición de coautores. Curiosamente este escenario lo dibujaba Walter Benjamin ya en 1934 en su obra Der author als produzent (El autor como productor):

"Estamos frente a una crisis de una forma de producción que se ha quedado obsoleta y anacrónica por los nuevos descubrimientos técnicos [...], ya que nos encontramos en medio de un proceso de transformación de las formas de gran alcance en el que muchos de los argumentos con los que solíamos trabajar podrían perder su poder, un momento dialéctico en el que la literatura gana en amplitud lo que pierde en profundidad, cuando la diferencia entre autor y público, mantenida artificialmente por la prensa, comienza a desaparecer, una época en la que el periódico está en un escenario de confusión, en la que, para bien o para mal, consumidores y lectores muestran signos de la impaciencia de quien se siente excluido, de quien piensa que tiene derecho a expresar sus intereses por sí mismo. Un escenario en el que la asimilación indiscriminada de los hechos va de la mano de una parecida asimilación indiscriminada de los lectores, que se ven a sí mismos instantáneamente elevados al nivel de coautores, en el que cabe preguntarse si se trata de una revolución o simplemente se alimenta un aparato de producción sin transformarlo, si el desafío es llevar consumidores a la producción [...] obteniendo coautores a partir de lectores o espectadores".

\section{La narrativa actual se caracteriza por la distribución multiplataforma y la multi- plicidad de autores}

Los cibermedios integran los lenguajes de todos los medios precedentes, tal como han puesto de manifiesto autores como Lev Manovich (2001). Además, como dice Henry Jenkins, "en la actualidad, los relatos más importantes tienden a fluir a través de múltiples plataformas de medios" (Jenkins, 2006, p. 46). En efecto, en el caso de la información de actualidad se produce el fenómeno de la distribución multiplataforma, que a veces da lugar a situaciones conflictivas. Ya en los inicios de la Web una resolución judicial guió la jurisprudencia de EUA y otros países en el caso The New York times vs Tasini et al. (533U.S 483, 2001). La Corte Suprema de los Estados Unidos ratificó la sentencia primera del Tribunal del Distrito de Nueva York por el que se condenaba a la empresa editora de The New York times a indemnizar a varios periodistas -encabezados por Jonathan Tasini- por haber publicado sus textos, cuyos derechos se habían adquirido para la edición impresa, también en un cd-rom.

Otra característica actual es la aceptación de contribuciones de los lectores a la hora de elaborar el discurso noticioso de los medios, satisfaciendo la necesidad ciudadana de participar en la construcción de la realidad social. Es lo que se ha denominado genéricamente como "periodismo ciudadano" (Deuze, 2008; Reich, 2008) y que algunos autores distinguen del "periodismo participativo" (Bruns, 2005; Singer et al., 2011), pese al consenso existente en equiparar ambos términos.

En el campo de la ficción es la fanfiction la manifestación más palmaria de la narrativa participativa: a partir de propuestas "de autor" como películas o series de televisión, los fans continúan la historia de múltiples maneras, proponiendo finales alternativos, desarrollando tramas o personajes y transformando la obra de muy diversas formas ${ }^{1}$. Se trata de prácticas -que también tienen antecedentes muy antiguos- que casi siempre son polémicas cuando no se tiene en cuenta al autor de la obra original. Recordemos las varias segundas partes del Lazarillo de Tormes, de muy distinto jaez y calidad literaria, o el caso bien representativo del Quijote de Alonso de Avellaneda, seguramente un seudónimo, cuya segunda parte, anterior a la del propio Miguel de Cervantes, provocó la ira de éste ante la "apropiación indebida" de sus personajes.

La regulación jurídica de los contenidos generados por los usuarios, los cambios en la estructura del mercado de la información -especialmente en los medios y grupos de comunicación-, así como la tendencia creciente a la convergencia mediática, han planteado nuevos problemas de propiedad intelectual de las obras informativas (y de ficción: Perryman, 2008). Si los usuarios adquieren la condición de autores de varios de esos contenidos-comentarios a textos, 
fotografías y vídeos-, y teniendo en cuenta la internacionalización y armonización de las normas jurídicas sobre propiedad intelectual y derechos de autor ${ }^{2}$, es indispensable abordar mediante un análisis jurídico comparativo (Doutrelepont, 1997; Reimann; Zimmerman, 2008) cuáles son las soluciones adoptadas para poder incluir dichos contenidos producidos por los usuarios -y especialmente aquellos que desarrollan la obra original- en el conjunto de la obra colectiva que son los medios de comunicación. Nuestra hipótesis principal es que, a mayor extensión de internet, más control tratan de ejercer las personas jurídicas en la adquisición y gestión de los derechos de explotación de las obras. En este mismo sentido se están llevando a cabo reformas legislativas que, además de armonizar sistemas jurídicos y leyes nacionales, acentúan la posibilidad de la cesión exclusiva de contenidos para su mejor posicionamiento y más fácil negociación de ulteriores sesiones a terceros, en un mercado cada vez más global.

Cada vez más las empresas tratan de ejercer un mayor control en la adquisición y gestión de los derechos de explotación de las obras

\section{Cuestiones de autoría}

La primera cuestión es comprobar cuáles son los cambios en la concepción de autoría que se están produciendo debido a la web 2.0 (o web social) y, en consecuencia, cuál es el régimen de propiedad intelectual que se aplica a los contenidos generados por el usuario y cómo se hace en las dos grandes tradiciones jurídicas: Common law o derecho anglosajón, y Civil law, continental o derecho de raíz latina. Si en el primer sistema el copyright hace residir su confianza en la empresa (la persona jurídica) como motor de la actividad intelectual, de información o de entretenimiento, el derecho de autor continental se basa más en la capacidad creativa de las personas (físicas) y, subsidiariamente, en la de las jurídicas (empresas) (Strowel, 1993).

Una de las principales diferencias que impidió una armonización anterior de las legislaciones de los países de ambos sistemas jurídicos es el reconocimiento de los derechos morales, que en nuestra tradición son derechos personales, irrenunciables e intransferibles, mientras que en los países del Common law -cuando se han reconocido a finales del siglo XX- ha sido con la particularidad de que se puedan ceder a la empresa, o mejor dicho, la empresa se puede subrogar en la condición de autor.

Puesto que tratamos de obras en colaboración (por tanto de autores múltiples) y de desarrollos narrativos posteriores (por tanto de obras derivadas), debe entenderse que la obligatoriedad de mencionar en todo momento a partir de qué obra original se produce la derivada es una exigencia no sólo legal, sino moral, exigible en todo momento. En cualquiera de los dos sistemas resulta obligado mencionar al autor material de la obra primera (una persona física, o varias si es una obra en colaboración de varios autores, hecho muy frecuente, pensemos en el caso de un reportaje, de una película o de una serie de televisión) y al lugar donde fue publicado, es decir, el periódico, cadena de televisión, etc., con lo que estaríamos mencionando también a la empresa o persona jurídica bajo cuya iniciativa se produjo la obra, y que en el caso de los países de Common law puede, mediante el mecanismo de subrogación mencionado, ser el autor de la obra ${ }^{3}$.

Cualquier autor tiene derecho a ser reconocido como autor de su obra y solicitar tanto reconocimiento moral como económico, si le corresponde (sobre la incidencia económica de los derechos morales, véase Wilkinson; Gerolami, 2009). En todo momento, con la ley en la mano, el periodista, el fotógrafo, el dibujante, el guionista de una serie tienen derecho a ser mencionados como autores de su obra.

El autor de los comentarios y otras aportaciones es aquel que los envía bajo su nombre -o con uno (o varios) seudónimos o nicknames (extremo también protegido por el derecho moral de autor: cómo firmar las propias obras)-. Estas aportaciones no sólo se producen en internet, sólo hace falta mirar la información meteorológica de muchas televisiones para comprobar que el público puede enviar de forma rápida, sistemática y con una calidad que muchas veces nada tiene que envidiar a la de los profesionales del medio audiovisual, fotos o vídeos que se muestran públicamente. La compensación, recordemos, no tiene por qué ser planteada en términos estrictamente monetarios, sino en otros con valor económico pero no directamente pecuniario, como el prestigio. En este sentido, es representativo el caso de Tasini $v$ AOL, en el cual Jonathan Tasini, ahora bloguero del Huffington post (que no remunera a quienes mantienen un blog en su sitio web), a quien el Tribunal del Distrito Sur de Nueva York negó el amparo que éste había solicitado al considerar que le correspondía, a él como al resto de firmas del Huffpost, un porcentaje de la venta del medio a America Online. Según Tasini, el interés de AOL por el Huffpost se debía, entre otras cosas, al prestigio de los blogueros.

\section{La justicia de los EUA no reconoció dere- chos económicos a los bloggers volunta- rios del Huffington post cuando éste fue comprado por $A O L$}

Frente a la doctrina del fair use de la Common law, el derecho civil continental propone la del "lucro cesante y el daño emergente" (uno pierde aquello que deja de ganar), de manera que, el hecho de que una obra derivada no tenga propósito de obtener una remuneración económica directa (Cabedo-Serna, 2011), no impide las ganancias de los autores de la obra original ahora o en el futuro. De hecho esa es la razón por la cual algunos autores muestran una intolerancia radical a las fanfictions derivadas de sus novelas -aunque han aceptado, mediante el correspondiente pago de derechos, la adaptación de las mismas al cine-. En otros casos se trata de reticencias como las de Joanne K. Rowling, autora de la saga de Harry Potter frente a las más de 61.000 secuelas que los lectores han ido colocando en internet, y en otros nos hallamos frente a cambios de criterio, como los de George Lucas respecto a las obras derivadas, en principio 
-pero no necesariamente-como mero divertimento sin ánimo de lucro de los fans de Star Wars.

Quizá no esté de más recordar que, aunque se disponga del permiso expreso del autor de la obra original para hacer una derivada (por ejemplo, la adaptación cinematográfica de una novela) las partes pueden pactar unos límites. Un caso bien aleccionador es la decisión final del Tribunal Supremo español, ratificando en 2006 sendas sentencias de 1998 del Juzgado de Primera Instancia de Madrid y de 2002 de la Audiencia Provincial de Madrid que daban la razón al escritor Javier Marías contra la directora Gracia Querejeta y su padre, el productor Elías Querejeta. Se condenaba a éstos a eliminar cualquier referencia a Javier Marías y al título de su novela Todas las almas en la película El último viaje de Robert Rylands (1996), al considerar Marías que la adaptación más que libre "libérrima" había desvirtuado

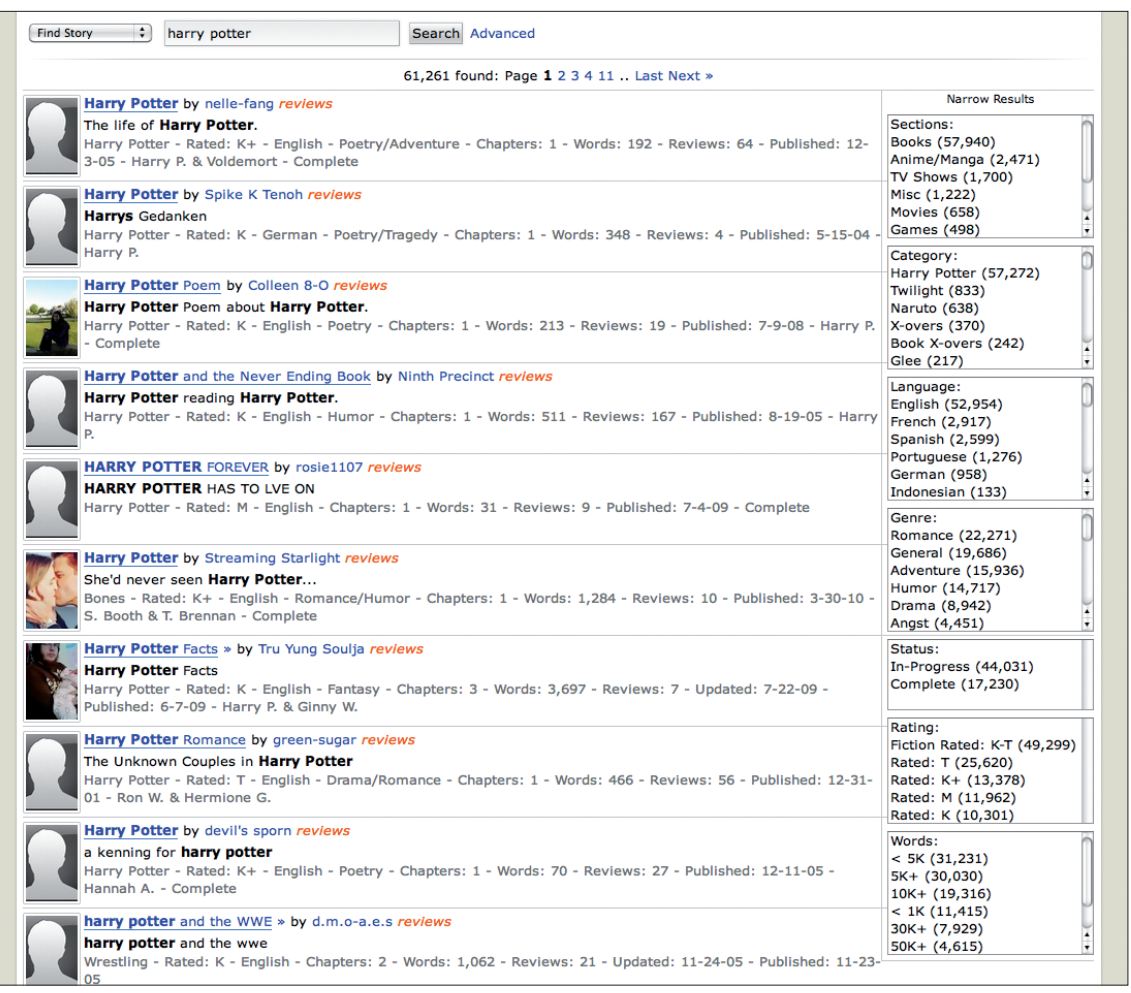

En Fanfiction se listan más de 61.000 historias derivadas de Harry Potter, http://fanfiction.net absolutamente todos sus personajes y tramas, cuando era una cláusula que el escritor se había cuidado de incluir en el contrato.

\section{Las normas y principios sobre derecho moral de autor también son de aplica- ción a los contenidos producidos por los usuarios}

A su vez, cuando hablamos de contenidos producidos por los usuarios, entendemos que las normas y principios sobre derecho moral de autor son igualmente de aplicación. Por tanto, quien comenta una noticia en una red social lleva a cabo un desarrollo argumental de su serie favorita, o contribuye con sus vídeos o fotos a enriquecer un foro de discusión, debe ser igualmente mencionado cuando exista algún tipo de retroalimentación: el medio debe citar esas obras, por muy menores o secundarias que se consideren. La cuestión de la originalidad de las obras, que aquí sólo podemos mencionar brevemente, es central para que pueda ejercerse la protección jurídica dispensada a sus autores, y no se refiere en términos jurídicos a su calidad, que no toca a los tribunales decidir, sino a que sea fruto del ingenio y el trabajo de sus autores.

\section{Tipos de obras}

Las leyes de propiedad intelectual protegen a cualquier persona que haya creado cualquier tipo de obra, siempre que sea original. Es este un extremo que contemplan todos los términos de uso de los cibermedios: la exigencia y el supuesto de que todo lo que envían los usuarios es original y disponen de los derechos sobre dicha obra, independientemente de su calidad (Örnebring, 2008). La responsabilidad que se derive del incumplimiento de este precepto recae en el usuario, no en el medio, que siempre se reserva el derecho de eliminar cualquier contenido que considere que no respeta este mandato legal.

Según las leyes de propiedad intelectual las obras pueden ser originales y derivadas (creadas a partir de las primeras). Hay muchos ejemplos de obras derivadas: las traducciones y los doblajes, por ejemplo, pero también supuestos que se apartan más de la obra original, como las adaptaciones o, en el caso de las narrativas transmediáticas o participativas de las que nos ocupamos, continuaciones de las obras originales, versiones alternativas, finales diferentes, spin offs, desarrollos de líneas argumentales secundarias, etc.

Las leyes de propiedad intelectual protegen a cualquier persona que haya creado cualquier tipo de obra, siempre que sea original

En cualquier caso, en puridad las obras derivadas sólo podrían producirse con el permiso expreso de los autores o de los cesionarios del derecho de transformación (en legislaciones como la española, en una doble vertiente de derecho moral y de derecho patrimonial o de explotación económica), lo que impediría, por otra parte, la extrema proliferación de fanfictions de algunas novelas o series famosas, de Harry Potter a Lost o Star Wars. La posibilidad de que el autor o sus derechohabientes impidan este tipo de obras existe tanto en el mundo jurídico anglosajón como en el continental, si bien, grosso modo, dependen de la tolerancia de dichos autores de la obra original o, en el mejor de los casos, del fair use. 
El fair use podría ser considerado, en términos jurídicos continentales, una figura que se añade a las excepciones legales (Tushnet, 2004, pp. 544, linea 36), si bien, al contrario de las que operan en la mayoría de las mencionadas explícitamente en los textos legales, se trata de una prueba a posteriori basada en la aplicación de la "regla de los tres pasos" reconocida por el Convenio de Berna para la protección de las obras literarias y artísticas de 1886, sucesivamente reforma$\mathrm{do}^{4}$, que en los EUA se ha convertido en cuatro reglas:

- regla transformativa, o propósito y carácter del uso;

- naturaleza de la obra original, que tenga derechos de autor o no, ya que si se trata de una obra en el dominio público ${ }^{5}$ sólo se perseguiría por parte de los poderes públicos una posible transgresión de los derechos morales, por ejemplo, la falta de atribución de paternidad a la obra original;

- que lo que utiliza la obra derivada sea una parte sustancial o no de la obra original;

- efecto sobre el mercado potencial, lo que equivaldría a nuestro ánimo de lucro.

El éxito del fair use ha hecho que, aunque sea ajeno a la concepción jurídica continental, sea invocado frecuentemente por la doctrina. Permite una cierta flexibilidad que va a favor de uno de los bienes jurídicos protegidos: junto a los derechos de los autores -el principal, a ganarse la vida dignamente mediante el sudor de su intelecto, excluyendo cualquier injerencia torticera por parte de cualquier otra persona- está el derecho a la cultura, un derecho, si se quiere, comunitario y que, aunque se olvida en ocasiones, estaba en el mismísimo título de la primera ley sobre derecho de autor, el Estatuto de la Reina Ana de 1710, o Act for the Encouragement of Learning (una reflexión actual sobre los derechos de los lectores puede hallarse en Litman, 2011).

Junto a la doctrina del fair use, en los países de raíz jurídica romana se invoca el principio del ánimo de lucro y la citada fórmula del "lucro cesante y el daño emergente". Strictu sensu: un autor podría impedir cualquier tipo de obra derivada de la suya original, incluso la fanfiction más amateur, argumentando que la existencia de un desarrollo ulterior de sus tramas y personajes impedirá que él mismo pueda eventualmente hacer lo propio, y obtener, igualmente de forma potencial, un beneficio económico. No obstante, la tendencia es a considerar que, si no hay un ánimo de lucro directo y presente, no debería aplicarse este principio.

Otro caso distinto es cuando se desarrollan personajes de obras que están ya en el dominio público, por haber pasado más de 70 años desde la muerte del autor ${ }^{6}$, aunque, en casos excepcionales como el de Peter Pan, cuyas regalías percibía -y percibe- una institución benéfica británica, se hayan extendido sucesivamente ${ }^{7}$. Aunque eso permitiría prolongar las narrativas de personajes cuyo autor haya muerto hace más de 70 años (un ejemplo sería la novela $A l$ morir don Quijote de Andrés Trapiello), cuando dicho personaje es, además, una marca registrada -como Mickey Mouse o cualquier otro personaje de la Disney- su utilización la impedirían no tanto las leyes de propiedad intelectual como las de propiedad industrial. Distinción propia de la Civil law, ya que en la Common law todo está dentro del sistema único de la propiedad intelectual en sentido anglosajón (véase nota 2).

\section{theguardian}

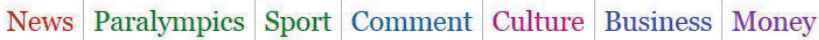
Business

\section{Never ends for Peter Pan}

JM Barrie bequeathed all rights to Peter Pan to Great Ormond Street hospital. Its imminent expiry is set to revive the debate over copyright term

\section{Katie Allen, media business correspondent}

guardian.co.uk, Friday 28 December 2007 12.16 GMT

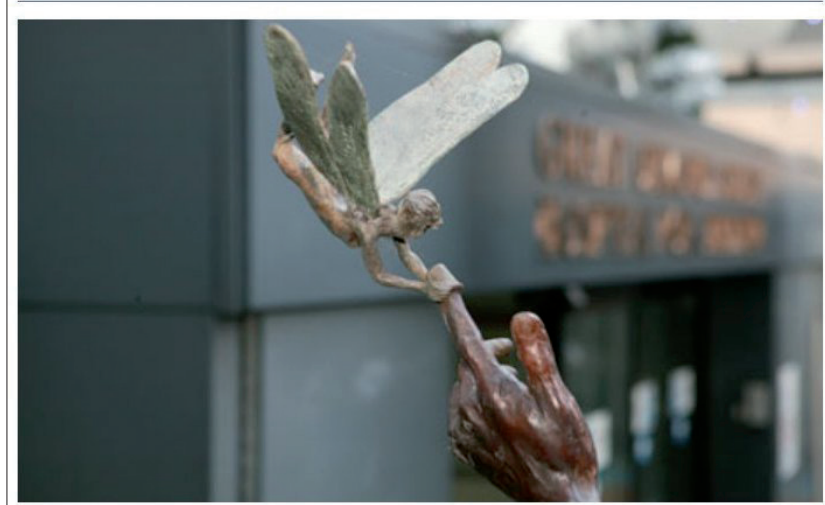

Detail from the Peter Pan statue outside Great Ormond Street hospital. Photo: Frank Baron

Artículo publicado en The guardian sobre los derechos de Peter Pan, diciembre de 2007.

http://www.guardian.co.uk/business/2007/dec/28/gtormondst

Por lo que respeta a las obras de no ficción, la mayoría de los avisos legales y términos de uso vetan explícitamente la posibilidad de hacer obras derivadas de los contenidos de su web, y ha de entenderse que esto incluye tanto los producidos por sus propios trabajadores como los enviados por los usuarios. Por ejemplo, hasta la $B B C$, que dispone de unas normas en cierto modo benévolas cuando se comparan con las de los medios privados, indica a los usuarios que "usted no puede crear trabajos derivados de BBC Online Services ni de $B B C$ Content ya sea en su totalidad o en parte, ni puede ayudar a nadie a intentarlo"8.

Un caso particular de obra derivada serían las parodias (véase Taktuf-Retif, 2009, referido a España), que mencionan o referencian de forma clara a un personaje. Aunque no son objeto de este breve texto, diremos que no dejan de provocar problemas legales cuando la parodia aprovecha el original para el lucro propio. Un ejemplo son los pastiches del Tintin de Hergé dibujados por Yves Rodier, que han alcanzado un notable éxito en internet. Otro caso paradigmático, al menos en el mundo anglosajón, es el de una parodia de una canción de Roy Orbison por parte del grupo 2Live Crew (Campbell v. Acuff-Rose Music, Inc., 510 U.S. 569, 1994) ${ }^{9}$, que no se consideró una infracción del derecho de autor. Pero la excepción de la parodia no sirve para cubrir cualquier supuesto de obra derivada, como se demostró en el caso Castle Rock Entertainment Inc. v. Carol Publishing Group acerca de la publicación de un juego sobe la serie Seinfeld ${ }^{10}$, o en el caso de una web sobre el léxico de Harry 
Potter, que fue llevado ante los tribunales, con éxito, por la autora J. K. Rowling (Warner Bros. Entertainment, Inc. v. RDR Books, No. 07 Civ 9667).

A veces, la obra derivada, permitida o no por el autor de la original, puede incurrir en plagio cuando hay falta de atribución por parte del autor de la obra derivada al autor de la obra original.

Aunque se trata de empeños amateur, obra de fans, el éxito de estas narrativas participativas es cuantitativamente enorme $^{11}$, lo que da visibilidad a la obra original pero también puede restarle usuarios. Existen, de hecho, varios conjuntos de recomendaciones jurídicas sistemáticas (Tushnet, 2011) a aquellos autores que quieran desarrollar con garantías una obra derivada de este tipo, como Copyright 101: a brief to copyright for fan fiction authors, de A. T. Lee ${ }^{12}$, o Copyright, work for hire, and other rights issues, en el sitio web Writers and editors ${ }^{13}$, y por supuesto en aquellos sitios web que son grandes repositorios de ficciones de este tipo, como Fanlore ${ }^{14}$.

Se trata de problemas que no se dan con tanta intensidad en el caso de la no ficción, pero que sí plantean algunas reflexiones en el caso de las informaciones de actualidad. Por muy exclusiva o scoop que sea una información conseguida por un medio de comunicación o un periodista, las excepciones legales que resultan de aplicación a las noticias de actualidad -ya desde el Convenio de Berna- permiten que otro profesional cualquiera pueda desarrollar la noticia si consigue nuevos datos, o mencionarla y rehacerla. Por supuesto, siempre citando autor y procedencia, como por ejemplo establece la Ley 1/1996 de Propiedad Intelectual española.

Las obras pueden ser: originales, derivadas, compuestas, en colaboración y colectivas

Junto a la obra derivada, nos encontramos también con otros tipos:

- obras compuestas: por aportaciones de varios autores;

- obra en colaboración: en régimen de coautoría;

- obras colectivas, que es el caso de los medios de comunicación (periódicos, revistas, webs, informativos de radio y televisión), cuya autoría corresponde excepcionalmente a una persona jurídica, la empresa bajo cuyo mandato y remuneración se crea la obra, y compuesta a su vez de obras individuales, y a veces derivadas.

Un caso particular de obra compuesta, muy típica de internet, es el mash-up, en el fondo otro tipo de obra derivada, un refundido de obras preexistentes. Para que sea una obra compuesta, y no en colaboración, es preciso que la obra nueva incorpore una obra ajena sin intervención (pero, obviamente, con el permiso) del autor de la obra preexistente. El ciberperiodismo está lleno de este tipo de obras. Una noticia a la que se le añaden enlaces que remite a noticias relacionadas, publicadas previamente por el medio, podría ser considerada una obra compuesta. Un especial que re- coge noticias, fotografías, vídeos, infografías sobre un tema concreto (por ejemplo, el campeonato mundial de fútbol, o unas elecciones), y que por tanto produce una obra nueva a partir de obras previas sería también una obra compuesta. Aquí hay que distinguir también dos niveles de autoría, el de las obras individuales que la obra nueva incorpora, que se respetan, y la autoría de la nueva obra, que para ser considerada como tal debe tener un mínimo de originalidad.

\section{Cesiones exclusivas y no exclusivas}

Cuando se produce una cesión en exclusiva del autor a otra persona (generalmente una empresa), siempre mencionando tanto su duración como el ámbito territorial, quiere decir que ésta ejercerá los derechos de explotación económica que le hayan sido cedidos con exclusión de cualquier otra persona. Cuando la cesión no es exclusiva, el autor puede explotar su obra también personalmente o cediendo los derechos a otra empresa. Por lo general, las empresas entienden que la cesión de los contenidos generados por los usuarios se les hace en los mismos términos de exclusividad con que tratan las obras de sus periodistas. Esta cesión en exclusiva suele también entender que incluye la libertad de publicación, negociación con terceras partes, e incluso modificación de la obra.

Ya no es infrecuente que los avisos legales de los cibermedios mencionen la cesión expresa de derechos de los lectores, y algunos como el portal brasileño Globo -uno de los conglomerados empresariales mediáticos más poderosos de América Latina-, ya mencionan que en caso de producirse algún tipo de beneficio económico por la explotación de estos contenidos, los autores de los mismos -comentarios, pero más frecuentemente vídeos, fotografías, textos enviados por los usuarios en el más puro estilo del periodismo participativo- tendrán derecho a percibir un porcentaje de los mismos. En realidad tampoco se trata de nada nuevo: en la prensa impresa pasaba lo mismo con las cartas al director, y es por tanto dudoso que el medio, o un periodista del mismo, puedan publicar una antología de esas cartas al director sin contar con el permiso expreso de sus autores.

La mayoría de los cibermedios se ocupan de adquirir, a título oneroso o no, la cesión en exclusiva de los contenidos que ofrecen. A título oneroso, mediante el pago de un sueldo, cuando son contratados (work made for hire, en el caso del sistema de Common (aw), o del correspondiente precio, cuando media un contrato mercantil o civil (en caso de los colaboradores o freelancers). A título no oneroso, cuando son los lectores quienes envían todo tipo de contenidos. En este segundo caso acostumbra a haber una cláusula mediante la cual, cuando los contenidos cedidos a título no exclusivo por los lectores ${ }^{15}$ se explotan de alguna manera -por ejemplo, una cesión a terceros- el autor pueda obtener una compensación equitativa por su esfuerzo. Todos emplean términos de uso o contratos que se ciñen a la normativa legal vigente, y todos sin excepción incluyen cláusulas jurisdiccionales atractivas: el usuario, sea de donde sea, sabe que se somete voluntariamente a la legislación sustantiva y procesal y a los tribunales del país de la razón social del medio al que está accediendo. 
La mayoría de medios ya dedican todo un apartado de su aviso legal a los contenidos producidos por los usuarios. Se incluyen tanto comentarios como servicios del tipo TimesPeople (en el caso del New York times) o incluso reader reviews, o críticas realizadas por los usuarios. Normas que en otros medios encuentran acomodo más como recomendaciones éticas, aquí son elevadas a la categoría de norma jurídica (contractual, por aceptación de dichas normas por parte del usuario). Además de las consabidas reglas de respeto, prohibición de enviar contenidos pornográficos, abusivos, xenófobos, etc., se establece muy claramente que habrá un propósito no comercial por parte del usuario. Pero, por el contrario, la empresa se asegura una cesión exclusiva de lo que envía el usuario mediante esta cláusula bien explícita:

"Usted concede al NYT una licencia perpetua, no exclusiva, mundial, libre de regalías, sublicenciable, que incluye el derecho ilimitado por parte de NYTimes.com o de cualquier otra parte designada por The New York Times de usar, copiar, transmitir, extractar, publicar, distribuir, exhibir o ejecutar públicamente, crear trabajos derivados, alojar, indexar, guardar en caché, etiquetar, codificar, adaptar y modificar (incluyendo el derecho de adaptar para streaming, descargar, radiodifundir, usar en dispositivos móviles, digitalizar, miniaturizar, escanear u otras tecnologías sin limitación) en cualquier formato o medio conocido actualmente o que se desarrolle en el futuro, las colaboraciones publicadas por usted en NYTimes.com o cualquier otro sitio web propiedad de The New York Times, incluyendo cualquier presentación publicada en NYTimes.com través de terceros" ${ }^{\prime 16}$.

Es una solución similar a la que aparece en el aviso legal de The guardian ${ }^{17}$ :

"Usted o el propietario del contenido todavía poseen los derechos de autor sobre el contenido que nos ha enviado, pero al enviárnoslo usted nos concede una licencia perpetua mundial incondicional, irrevocable, no exclusiva, libre de regalías, totalmente transferible, perpetua en todo el mundo para utilizar, publicar o transmitir, o para autorizar a terceros a utilizar, publicar o transmitir su contenido en cualquier formato y en cualquier plataforma, ya sea conocido ahora o se invente más adelante".

Es decir, mientras que por un lado el medio se hace con la posibilidad de cesión a terceros de todos los contenidos que le son remitidos, de alguna manera (en este caso, tácita) se garantiza al lector que se gestionarán adecuadamente dichos contenidos, incluso cuando se negocie con terceras personas jurídicas. Según está redactado parece un acuerdo leonino.

Los hay más abiertos. Un modelo más permisivo es el de la $B B C$, que en su web invita a los usuarios a "hacer, compartir o presentar sus propias contribuciones originales a BBC Online Services" mientras que establece a modo de contrato en las normas contenidas en los Terms of use "cómo puede hacerlo y cómo la $B B C$ puede utilizar su contribución", la cual "puede incluir texto, fotografías, gráficos, vídeo o audio".

En concreto, la $B B C$ propone al lector hacer un uso no venal de sus contenidos, lo que es común en todos los cibermedios (aunque habría que ver qué ocurre cuando la aportación del usuario se añade o completa una noticia de la versión Premium y, por tanto, de pago), a cambio de garantizarle que los moderará y hará un uso racional de los mismos, siempre -esto es común en todos los medios, por razones obvias de evitar cualquier tipo de implicación penal- reservándose el derecho a recortarlos o incluso eliminarlos si estima que vulneran las normas éticas contenidas en el mismo aviso legal o que no se trata de contenidos originales, sino de plagios. En concreto, la $B B C$ garantiza que:

“los derechos de autor de su contribución seguirán siendo de usted y este permiso no es exclusivo, por lo que usted puede seguir utilizando libremente el material que nos ha proporcionado, e incluso permitir que otros lo usen".

Ello va más allá de un mero reconocimiento de paternidad (derecho moral), que, aunque sea posible en el sistema de copyright de la Common law, en ningún caso se estima cedido (si bien también se deja claro que "normalmente mostraremos su nombre con su contribución, a menos que usted solicite lo contrario, pero por razones operativas esto no siempre es posible", no se especifican las razones operativas que impedirían ejercer el derecho a ser reconocido como autor de la aportación enviada a la $B B C$ ), e incluye también los derechos de explotación económica de las obras, según la vigente Copyright Act británica de 1988.

Hay pocas excepciones. Eskup, de El país (no así el sitio web completo del diario) emplea Creative commons, un sistema de licencias que permite a cualquier usuario y a cualquier autor definir con claridad qué quiere ceder o no y con qué alcance. Ahora bien, y muy significativamente: mientras que la licencia Creative commons empleada en Eskup ("Reconocimiento -No comercial- Sin obras derivadas 3.0") permite "copiar, distribuir y comunicar públicamente los contenidos de nuestra web", se preocupa de dejar claro que "no se pueden hacer obras derivadas, ni hacer un uso comercial en internet de esos contenidos". La licencia ni siquiera se aplica, "no al menos directamente", "a los contenidos de los usuarios, cuyos derechos quedan estrictamente reservados a su titular", cuyo consentimiento es necesario para cualquier acción transformativa (tal como, por otra parte, establece la Ley de Propiedad Intelectual española, 1/1996) ${ }^{18}$.

http://eskup.elpais.com

Sin embargo, la postura de los medios de comunicación, al menos en EUA, es favorable a una cierta tolerancia a las obras derivadas, siempre que no causen daños económicos graves (Tushnet, 2004). En un amici curiae (un escrito espontáneamente dirigido a un tribunal mientras éste examina una causa, en concreto una relacionada con el tema que nos ocupa, la del escritor J. D. Salinger contra Colting et al.), New York Times, Associated Press, Gannett y Tribune Company dirigieron al Tribunal de Apelaciones de los EUA un escrito en el que, entre otras cosas, afirmaban que:

"Amici [se refieren a ellos mismos] publican todos los días material con copyright, y dependen de la ley de copyright para proteger sus escritos. La necesidad de protección de los derechos de autor es hoy más intensa que nunca, ya que las tecnologías digitales hacen cada vez más fácil que terceros puedan aprovechar y reutilizar los frutos del costoso esfuerzo de recopilar noticias. Sin embargo, Amici 
creen firmemente que una medida cautelar de la ley de copyright no puede prevalecer sobre las prerrogativas de la Primera Enmienda [que regula la libertad de expresión], y que no puede tolerarse la prohibición de hacer obras transformativas".

Los contenidos aportados por el usuario se consideran cedidos en régimen no exclusivo, y pueden ser explotados por sus autores en cualquier otro medio y formato

\section{Conclusiones}

El examen somero de las principales cuestiones jurídicas que afectan a la narrativa y los contenidos producidos por el usuario nos lleva a plantear algunas conclusiones, a partir de la constatación de que, tanto en el ámbito de la ficción como de la no ficción, la tendencia a la participación del usuario en régimen de autoría o coautoría es un fenómeno en crecimiento:

- El carácter de obra derivada se impone al de obra en colaboración u obra colectiva, lo que se aprecia claramente en contenidos supuestamente colaborativos como los de la red social Eskup de El país. Ello es todavía más claro en el caso de la ficción, donde las líneas narrativas se expanden a través de, por ejemplo, fanfictions.

- En prácticamente todos los medios examinados, los contenidos de los usuarios se tratan de forma independiente de los contenidos producidos por el propio medio. Aunque el medio deje claro que los derechos de explotación de forma colectiva de todos los contenidos que publican les pertenecen, es recomendable dar a los contenidos que provienen de fuera de la redacción un tratamiento jurídico diferenciado.

- En general, y al contrario de lo que ocurre con los contenidos generados por los profesionales, los aportados por el usuario se consideran cedidos en régimen no exclusivo, y por tanto pueden ser explotados por sus autores en cualquier otro medio y formato.

- Los contenidos generados por los usuarios se consideran realizados sin ánimo de lucro y tampoco el medio pretende obtener beneficio económico directo de ellos -aunque esto sería algo más discutible en el caso de los medios informativos-. Como la gestión de todos los contenidos, generados por el usuario o no, se considera cedida al medio que los publica, en caso de ulteriores ganancias por cesión a terceros, el usuario debe ser compensado por ello.

- En el ámbito anglosajón, la ausencia de ánimo de lucro y el fair use (éste cada vez más invocado por la doctrina continental) son considerados imprescindibles para que los autores de la obra original muestren una tolerancia -a la que no están obligados por ley- hacia las obras derivadas, que, si bien pueden distraer en cierto modo la atención hacia la obra original que desarrollan, por otro lado le otorgan prestigio, lo que no deja de ser una justa compensación.

\section{Notas}

1. Existe incluso una organización dedicada a las obras derivadas: Organization for Transformative Works. Tienen también una revista, Transformative work and cultures.

http://transformativeworks.org

http://journal.transformativeworks.org/index.php/twc

2. La mayoría de las leyes que tratan de los derechos de los autores -y sus cesionarios- son denominadas en el Common law como Copyright acts, pero es obvio que los derechos reconocidos van más allá del mero derecho de copia, llamado en la tradición jurídica continental "derecho de reproducción", pensado para las obras impresas (cuyos ejemplares físicos deben ser luego distribuidos, lo que da lugar al "derecho de distribución"). A su vez, las leyes que versan sobre esos derechos, a los que se han ido añadiendo los que tratan de dar solución a la comunicación pública de productos audiovisuales, por ejemplo, son denominados en el derecho continental de propiedad intelectual. Por si fuera poca la aparente confusión terminológica, en los países de Common law se entiende por intellectual property no sólo el copyright, sino también el sistema de protección jurídica a patentes y marcas, que en cambio, en los países de derecho continental, son protegidos mediante las leyes de propiedad industrial, relacionadas pero con un sistema y grado de protección diferente a las de propiedad intelectual. Por todo ello, la doctrina prefiere sistemáticamente el término derecho(s) de autor para referirse a las cuestiones que tratamos aquí.

3. Esta tendencia se aprecia también en la creciente importancia que las leyes de propiedad intelectual continentales, -por ejemplo la española tras la reforma de 2006- conceden a las obras colectivas realizadas por iniciativa de una persona jurídica, como las publicaciones periódicas.

\section{4. http://www.wipo.int/treaties/es/ip/berne/trtdocs_ wo001.html}

5. Las obras en dominio público, que al contrario que las huérfanas sí tienen autor conocido (y por tanto, al menos en la tradición jurídica continental, mantienen los derechos morales), son aquellas sobre las cuales no hay derechos de disfrute exclusivos patrimoniales, bien porque su autor ha fallecido hace más de setenta años (según la doctrina continental, adoptada con reticencias y particulares adaptaciones por los países de Common law, sirva de ejemplo la Sonny Bono Act estadounidense, también llamada peyorativamente Mickey Mouse Act, porque alargaba el disfrute de los derechos de las obras justo cuando las primeras películas del famoso personaje de Disney, producidas en 1923, estaban a punto de entrar en el dominio público); bien porque -esto en principio sólo es aplicable a determinados países, generalmente los de Common law- su autor así lo dispone en vida, decisión que se entiende no revocable; bien, y esto es una particularidad del sistema de Common law, porque la obra no se había registrado, requisito indispensable hasta hace relativamente poco tiempo para que alguien reclame derechos sobre ella, al contrario de lo que ocurre en el derecho continental, donde por lo general, como en el caso de España, el registro tiene valor declarativo pero no constitutivo del derecho sobre la obra. 
6. Con algunas excepciones: por ejemplo, los derechos patrimoniales sobre aquellas obras producidas mientras estaba en vigor la anterior Ley de Propiedad intelectual española, la de 1987, tienen una duración de 80 años después de la muerte del autor.

7. Se trata, en cualquier caso, de una especie de derecho de autor perpetuo excepcional y limitado a la percepción de regalías por determinados conceptos (representación pública, publicación y, lo que resulta más interesante para este artículo, la adaptación), en ningún caso el Hospital for Sick Children of Great Ormond Street se subroga en la condición de autor. Las sucesivas extensiones de este derecho excepcional en la Copyright Act británica de 1988 (curiosamente, el copyright sobre Peter Pan hubiese expirado el 31 de diciembre de 1987, como recoge el artículo 131 de esa ley), cada vez que la obra estaba a punto de pasar al dominio público, la última en 2008 , no han estado exentas de polémica (véase, por ejemplo, el reportaje de The guardian), y en ningún caso obligan a aquellos países cuya legislación indica que los derechos sobre obras extranjeras se aplican en función del ordenamiento del país donde se explota la obra. Concretamente, en EUA la obra de Barrie ya está en el domino público e incluso Disney Corporation, que habitualmente se había mostrado partidaria de la extensión de los derechos a recibir regalías por parte del Hospital, como lo era a extender los derechos sobre sus propias creaciones, y durante mucho tiempo había sido cesionaria de los derechos sobre la animación de Peter Pan, por tanto, derechos de transformación de la obra, en 2004 publicó en EUA una secuela, Peter and the Starcatchers, sin devengar regalías al Hospital for Sick Children inglés.

http://www.guardian.co.uk/business/2007/dec/28/gtor mondst

\section{8. $h t t p: / / w w w . b b c . c o . u k / t e r m s$}

9. El Tribunal Supremo estadounidense revocó la decisión de un tribunal de primera instancia que había dictaminado que la parodia realizada por 2Live Crew de la canción "Oh, pretty woman" de Roy Orbison constituía una infracción del derecho de autor.

10. Se sentenció que Carol Publishing Group había infringido el copyright al vender un trivial basado en la serie $\mathrm{Se}$ infeld, producida por Castle Rock Entertainment. El tribunal rechazó el fair use esgrimido por la parte demandada considerando que en el trabajo derivado el propósito transformador era "mínimo o inexistente" (haciendo referencia al fallo del antes citado caso Campbell v Acuff-Rose Music, Inc., de 1994).

11. Para calcular el enorme potencial de estas narrativas de ficción, consultar Fan Fiction, probablemente el repositorio más grande de estas obras derivadas en la Web.

http://fanfiction.net

\section{2. http://www.whoosh.org/issue25/lee1.html}

13. http://www.writersandeditors.com/copyright_work for_hire_and_other_rights_issues_57380.htm

14. http://fanlore.org/wiki/Professional_Author_Fanfic_ Policies

15. No se impide la explotación paralela o posterior de dichos contenidos, de nuevo en régimen no exclusivo, en otros medios o en antologías (lo que constituye el derecho de colección en la normativa española de propiedad intelectual).

16. http://www.nytimes.com/content/help/rights/terms/ terms-of-service.html

\section{7. http://www.guardian.co.uk/help/terms-of-service}

18. http://eskup.elpais.com/Estaticas/aviso_legal.html http://eskup.elpais.com/Estaticas/creative_commons.html

19. Este artículo es resultado del proyecto "Audiencias activas y periodismo. Interactividad, integración en la web y buscabilidad de la información periodística". CSO201239518-C04-02. Plan Nacional de I+D+i, Ministerio de Economía e Innovación. Investigador principal: Lluís Codina.

\section{Bibliografía}

Aarseth, Espen. Cybertext: perspectives on ergodic literature. Baltimore; London: The Johns Hopkins University Press, 2007. ISBN: 9780801855795

Bal, Mieke. Narratology. Introduction to the theory of narrative. Third edition. Toronto: University of Toronto Press, 2009. ISBN: 9780802078063

Benjamin, Walter. El autor como productor (1934). Trad. de Jesús Aguirre, Taurus Ed., Madrid, 1975.

Bruns, Axel. Gatewatching: collaborative online news production. Peter Lang: New York, 2005. ISBN: 9780820474328

Cabedo-Serna, Llanos. El derecho de remuneración del autor. Madrid: Dykinson, 2011. ISBN: 9788499822099

Deuze, Mark. "The professional identity of journalists in the context of convergence culture". Observatorio, 2008, v. 4, n. 2, pp. 103-117.

http://www.obs.obercom.pt/index.php/obs/article/view Article/216

Deuze, Mark; Bruns, Axel; Neuberger, Cristoph. "Preparing for an age of participatory news". Journalism practice, 2007, v. 1, n. 3, pp. 322-338.

http://eprints.qut.edu.au/9785

Doutrelepont, Carine. Le droit moral de l'auteur et le droit communautaire: analyse en droit comparé et en droit européen. Bruxelles, Paris: Bruylant, 1997. ISBN: 978 2802706427

Evans, Elisabeth. Transmedia television: audiences, new media and daily life. New York: Routledge, 2011. ISBN: 978 0415882927

Hausken, Liv. Textual theory and blind spots in media studies. En: Ryan, Marie-Laure. Narrative across media. The languages of storytelling. Lincoln; London: University of Nebraska Press: 2004, pp. 391-403. ISBN: 9780803289932

Jenkins, Henry. "The cultural logics of media convergence". International journal of cultural studies, 2004, v. 7, n. 1, pp. 33-43.

http://ics.sagepub.com/content/7/1/33.full.pdf

http://dx.doi.org/10.1177/1367877904040603

Jenkins, Henry. Convergence culture. Where old and new 
media collide. New York: New York University Press, 2006. ISBN: 9780814742815

Litman, Jessica. "Readers' copyright". Journal of the Copyright Society of the USA, Winter, 2011, pp. 1-28.

http://papers.ssrn.com/sol3/papers.cfm?abstract_id= 1774932

Manovich, Lev. The language of new media. Cambridge, MA: The MIT Press, 2001.

http://www.manovich.net/LNM/Manovich.pdf

Örnebring, Henrik. "The consumer as producer of what? User-generated tabloid content in The sun (UK) and Aftonbladet (Sweden)". Journalism studies, 2008, v. 9, n. 5, pp. 771-785.

http://jclass.umd.edu/classes/jour698m/ornebring.pdf http://dx.doi.org/10.1080/14616700802207789

Page, Ruth; Bronwen, Thomas (eds.). New narratives: stories and storytelling in the digital age. Nebraska: University of Nebraska Press, 2011. ISBN: 9780803217867

Page, Ruth. New perspectives on narrative and multimodality. London: Routledge, 2010. ISBN: 9780415516563

Passalacqua, Franco; Pianzola, Federico. "Defining transmedia narrative: problems and questions. Dialogue with Mary-Laure Ryan". Enthymema, 2011, n. 4, pp. 65-71. http://riviste.unimi.it/index.php/enthymema/article/ view/1188

http://dx.doi.org/10.6092/2037-2426/1188

Perryman, Neil. "Doctor Who and the convergence of media". Convergence. The international journal on research into new media technologies, 2008, v. 14, n. 1, pp. 21-39. http://con.sagepub.com/content/14/1/21.full.pdf+html http://dx.doi.org/10.1177/1354856507084417

Reich, Zvi. "How citizens create news stories". Journalism studies, 2008, v. 5, n. 9, pp. 739-758. http://dx.doi.org/10.1080/14616700802207748

Reimann, Mathias; Zimmermann, Reinhard (cords). The Oxford handbook of comparative law. Oxford: Oxford University Press, 2008. ISBN: 9780199296064

Ryan, Marie-Laure (ed.). Narrative across media. The languages of storytelling. Lincoln; London: University of Nebraska Press, 2004. ISBN: 9780803289932

Singer, Jane B. et al. Participatory journalism: guarding open gates in online newspapers. New York: Wiley-Blackwell, 2011. ISBN: 9781444332278

Strowel, Alain. Droit d'auteur et copyright: divergences et convergences: étude de droit comparé. Bruxelles, Paris: Bruylant, 1993. ISBN: 9782802708537

Taktuf-Retif, Aurora-Marlene. El derecho de transformación. Especial referencia a la parodia. Tesis doctoral. Madrid: Universidad Carlos III, 2009.

http://e-archivo.uc3m.es/bitstream/10016/7355/1/ aurora_tactuk_tesis.pdf

Tushnet, Rebecca. "Legal fictions: copyright, legal fiction, and a new common law". Loyola of Los Angeles entertainment law journal, 1997, v. 17, n. 3, pp. 651-686.

http://www.tushnet.com/legalfictions.pdf

Tushnet, Rebecca. "Copy this essay: how fair use doctrine harms free speech and how copying serves it". The Yale law journal, 2004, v. 114, pp. 535-590.

http://www.tushnet.com/copythisessay.pdf

Wilkinson, Margaret-Ann; Gerolami, Natasha. "The author as agent of information policy: The relationship between economic and moral rights in copyright". Government information quarterly, 2009, v. 26, n. 2, pp. 321-332.

http://dx.doi.org/10.1016/j.giq.2008.12.002

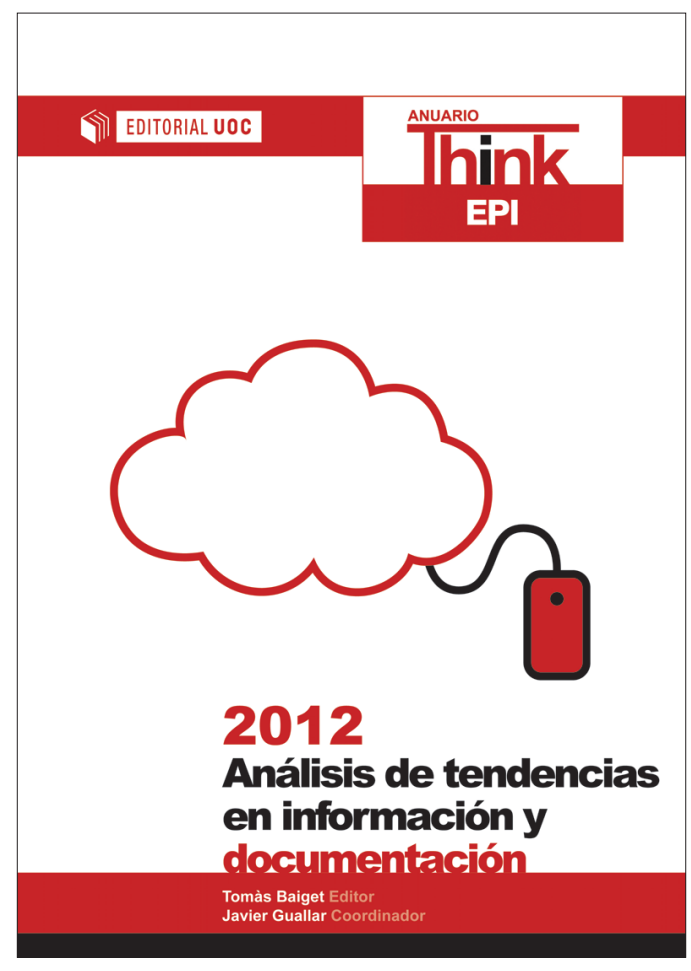

\section{Anuario ThinkEPI 2012}

Anuario científico-técnico que incluye 44 notas de análisis producidas por el think tank Grupo ThinkEPI, 10 informes de situación, y una selección de reseñas de informes técnicos anuales de todo el mundo.

Anuario ThinkEPI, v. 6, Barcelona: EPI SCP; Editorial UOC, 2012, 340 pp.

ISSN: 1886 6344; ISBN: 9788497889858

Información y adquisición en:

http://www.thinkepi.net/anuario-thinkepi/ anuario-thinkepi-2012

$\mathrm{y}$

http://www.editorialuoc.cat/anuariothinkepi 2012-p-985.html 